\title{
Using fMRI to Quantify the Time Dependence of Remifentanil Analgesia in the Human Brain
}

\author{
Richard G Wise*,1,2, Pauline Williams ${ }^{3}$ and Irene Tracey ${ }^{1,2}$ \\ 'Department of Clinical Neurology, Centre for Functional Magnetic Resonance Imaging of the Brain (FMRIB), University of Oxford, John Radcliffe \\ Hospital, Oxford, UK; ${ }^{2}$ Department of Human Anatomy and Genetics, University of Oxford, South Parks Road, Oxford, UK; ${ }^{3}$ GlaxoSmithKline, \\ New Frontiers Science Park, Third Avenue, Harlow, Essex, UK
}

\begin{abstract}
To understand and exploit centrally acting drugs requires reliable measures of their time course of action in the human brain. Functional magnetic resonance imaging ( $\mathrm{fMRI}$ ) is able to measure noninvasively, drug-induced changes in task-related brain activity. Here, we have characterized, in a specific region of the brain, the time of onset of action and the half-life of action of a clinically relevant dose of a potent opioid analgesic agent, remifentanil. These times were established from the temporal variation of the amplitude of the blood oxygen level-dependent response in the insular cortex contralateral to a painfully hot thermal stimulus, in volunteers receiving a remifentanil infusion. The insular cortex has repeatedly been reported as activated by noxious thermal stimulation. The times of onset and offset of drug action were each characterized by a half-life for changes in $\mathrm{MRI}$ signal from within the insula. These characteristic times agreed with the observed drug-induced analgesia and previous pharmacokinetic-pharmacodynamic measurements for remifentanil. We have successfully measured, for the first time using $\mathrm{fMRI}$, temporal pharmacological parameters for a CNS-active drug based on its effect on task-related activity in a specific brain region. Comparison of the time course of regional brain activity with pain perception could reveal those regions engaged in drug-induced analgesia.

Neuropsychopharmacology (2004) 29, 626-635, advance online publication, I 5 December 2003; doi: I 0.I 038/sj.npp. I 300364
\end{abstract}

Keywords: pain; analgesia; fMRI; remifentanil; pharmacokinetics; pharmacodynamics

\section{INTRODUCTION}

Functional neuroimaging techniques such as functional magnetic resonance imaging (fMRI) have made it possible to study in vivo drug-induced changes in regional brain activity (Leslie and James, 2000; Stein, 2001; Tracey, 2001). In pharmacological fMRI studies to date, the temporal profile of the drug's effect has been assumed. The assumption has either been implicit in comparing fMRImeasured brain activity before and after administration (Breiter et al, 1997; Coull et al, 2001; Furey et al, 2000; Hariri et al, 2002; Honey et al, 1999; Kimberg et al, 2001; Kleinschmidt et al, 1999; Mattay et al, 2000; Thiel et al, 2001, 2002), or the assumption has been more explicit in incorporating pre-existing pharmacokinetic parameters into the analysis of fMRI data (Stein et al, 1998; Bloom et al, 1999; Wise et al, 2002). In either case, the aim has been

*Correspondence: RG Wise, Centre for Functional Magnetic Resonance Imaging of the Brain, University of Oxford, John Radcliffe Hospital, Oxford OX3 9DU, UK, Tel: + 44-1865-222725, Fax: + 44|865-2227|7, E-mail: wise@fmrib.ox.ac.uk

Received 10 June 2003; revised 07 October 2003; accepted 29 October 2003

Online publication: 20 October 2003 at http://www.acnp.org/citations/ Npp I030030359/default.pdf to simply identify drug-induced changes in brain activity. fMRI must be developed beyond such studies if it is to be valuable for pharmacological assays. The development and assessment of novel centrally acting drugs requires a better understanding of their sites, mechanisms and time-courses of action. We suggest that fMRI has the potential to measure the regional time dependence of drug activity of certain compounds in the brain. This paper demonstrates that potential for the first time using painful thermal stimulation and remifentanil, a potent, short-acting, $\mu$-selective opioid analgesic agent. The opportunity afforded by fMRI to compare the time-courses of drug effects in different brain regions with subtle behavioral changes, such as analgesia, could help to establish the networks critical for analgesia and hence the brain systems to be targeted for maximum therapeutic effect.

The rates of onset and offset of action of a drug are clinically important. They depend on the mechanism and site of action as well as the temporal variation of the drug concentration in the body, described by the drug's pharmacokinetics. The delay in onset of a centrally acting drug once in the blood can arise from the time taken for a significant concentration to be achieved at the notional effect site of the drug, for example brain. The time dependence of drug action may be measured from the 
effects of the drug on the body, known as the pharmacodynamics. In the case of remifentanil, pain threshold (Glass et al, 1993) has been used to characterize the time dependence of the analgesic effect. Additionally, less analgesia-relevant measures have been employed including spectral characteristics of the electroencephalogram (Egan et al, 1996; Hermann et al, 1999; Hoke et al, 1997; Minto et al, 1997a, b), which provides a measure of brain activity, but is not spatially localized, requires doses beyond the normal clinical range and more importantly is not directly associated with pain processing. PET can map the concentration or binding of pharmacological agents over time (Malizia et al, 1996; Pappata et al, 1996) or measure task or drug-induced changes in cerebral blood flow (Petrovic et al, 2002). However, PET is restricted as a tool for examining task-related brain activity by its relatively long measurement time and invasiveness. fMRI, however, noninvasively provides better spatial and temporal localization of brain activity during complex event-related tasks such as thermal pain (Peyron et al, 2000) that may be modulated by analgesic drugs including opioids (Tracey et al, 2000b; Tracey, 2001). We have previously identified from fMRI signal, remifentanil-induced regional modulation of pain-related brain activity (Wise et al, 2002) with the help of a model for drug effect site concentration based on EEG measurements (Minto et al, 1997a,b). The study presented here considerably extends this work by characterizing the time-course of drug effect within the brain using fMRI. We examine the temporal variation of thermal pain-related brain activity in the insular cortex during infusion of remifentanil. This is then used, for the first time, to calculate pharmacokinetic-pharmacodynamic parameters describing the time of onset and offset of drug action based on changes in pain-related brain function. Our fMRI measures are supported by their agreement with the time-course of reported analgesia during this study and previous crude EEG measurements (Minto et al, 1997a,b).

\section{MATERIALS AND METHODS}

The study was approved by the Oxfordshire Clinical Research Ethics Committee and volunteers gave informed written consent. Nine healthy male volunteers (mean age $\pm S D, 29 \pm 9$ years) were examined on three different occasions. The first examination was a medical screen to ensure that volunteers tolerated remifentanil, determined by an anesthetist monitoring blood pressure and oxygen saturation. The second and third examinations, randomized in order across subjects were (a) fMRI combined with painful stimulation and a remifentanil infusion to a maximum estimated plasma concentration of $1.0 \mathrm{ng} / \mathrm{ml}$ and (b) fMRI examination with painful stimulation and a saline infusion volumetrically equivalent to that of the remifentanil infusion.

\section{Painful Thermal Stimulation}

During each functional imaging session, 28 noxious thermal stimuli each of $3 \mathrm{~s}$ duration were applied to the dorsum of the left hand (Figure 1). A purpose built, MR compatible thermal resistor $(1.5 \times 2 \mathrm{~cm})$ delivered the fast ramping $(30$ -

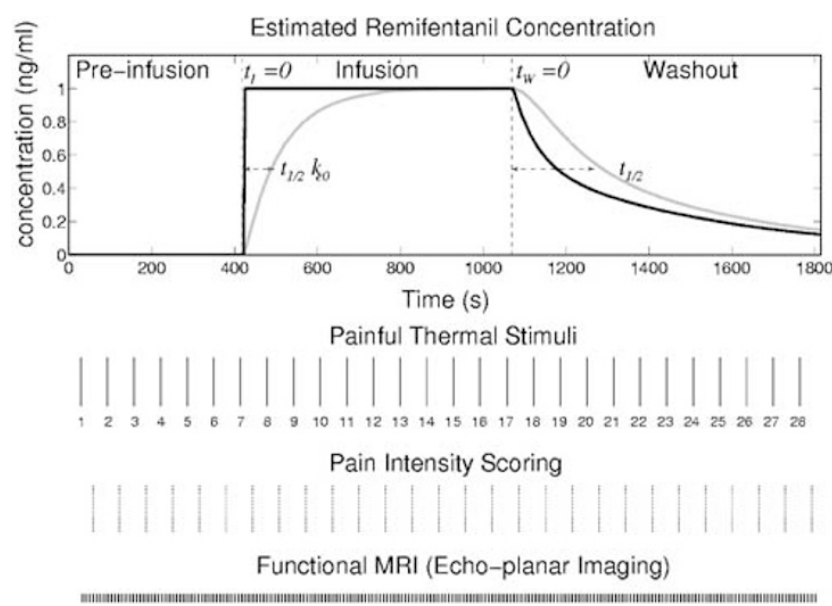

Figure I One imaging session lasting approximately $30 \mathrm{~min}$. Intravenous infusion was performed with either remifentanil or saline. During saline infusions, remifentanil concentration remained zero. Painful thermal stimuli were of a constant temperature within one session. The heavy line in the plot indicates the estimated blood plasma remifentanil concentration. The half-tone line indicates the effect site concentration of remifentanil estimated from the pre-existing pharmacokinetic model based on EEG measurements (Minto et al, 1997a, b). The half-life of equilibration between the plasma compartment and the effect site $\left(t_{1 / 2} k_{e}\right)$ and the washout halflife of the effect site concentration $\left(t_{1 / 2}\right)$ are shown schematically.

$60^{\circ} \mathrm{C}$ in $0.8 \mathrm{~s}$ ) painful thermal stimuli. This allowed a short stimulus to be delivered for event-related fMRI studies. Three seconds was an appropriate duration for a stimulus that was safe and immediately felt as hot without a significant initial component of warmth. The $3 \mathrm{~s}$ thermal stimulus has been shown in our previous studies of remifentanil (Tracey et al, $2000 \mathrm{~b}$ ) to give robust pain-related activity, which could be scored by volunteers for pain intensity. A brief stimulus is also advantageous to avoid significant change in remifentanil concentration during the stimulus itself. The mean interstimulus interval was $65.3 \mathrm{~s}$. There was a pseudorandom sequence of interstimulus intervals approximately uniformly distributed in the range $61.5-70.5 \mathrm{~s}$. This variation in interstimulus interval was adopted to reduce regularity of stimulus presentation and hence reduce the volunteers' ability to predict the arrival of the next stimulus. A relatively long interstimulus interval was employed to allow time for reporting of perceived pain intensity between stimuli and to reduce sensitization of the volunteer to the stimulus. Thermal stimuli delivered too rapidly in succession to a site on the skin may result in changes in perceived intensity over time of identical stimuli.

To improve the effective temporal sampling of the blood oxygen level-dependent (BOLD) hemodynamic response to the painful stimuli, consecutive noxious stimuli were offset in time by a whole number of interscan (TR) periods plus $1 / 2 \mathrm{TR}=1.5 \mathrm{~s}$. Before imaging while subjects lay in the MRI scanner, the thermal pain intensity threshold was determined for each subject. The stimulus temperature was adjusted iteratively to elicit a consistent rating of 3-4 indicating moderate-strong pain on a 5-point numerical rating scale (Jensen and Karoly, 2001). That temperature was used for each stimulus during the functional imaging session. During imaging, $21 \mathrm{~s}$ after the end of each thermal stimulus (Figure 1), the 5-point numerical pain intensity scale was shown to the subjects for $5 \mathrm{~s}$. This prompted the 
subjects to report the most recent stimulus for pain intensity by pressing one of five buttons, with the right hand. A pain scale of 5 points, eliciting a button press, was chosen because of the need to perform and record pain rating repeatedly and rapidly to provide minimal perturbation in the fMRI data associated with scoring.

\section{fMRI}

Imaging was performed at $3 \mathrm{~T}$ with an Oxford Magnet Technology, $1 \mathrm{~m}$ bore magnet. The magnet was driven by a Varian Unity Inova console. Whole-brain gradient-echo echo-planar imaging (EPI) was performed giving $\mathrm{T} 2 *$ weighting or BOLD contrast (image matrix $64 \times 64$; $4 \times 4 \mathrm{~mm}$ pixels; echo time (TE) $30 \mathrm{~ms}$ ). Each volume comprised 21 contiguous axial slices, $6 \mathrm{~mm}$ thick. Volumes were acquired continuously with a repetition time (TR) of $3 \mathrm{~s}$. For each subject, a T1-weighted structural scan (64 contiguous $3 \mathrm{~mm}$ axial slices; in-plane field of view $256 \mathrm{~mm}$, $1 \times 1 \mathrm{~mm}$ pixels) was acquired. This was used for anatomical overlay of brain activation and to assist in placing individual subject's data into a common stereotactic space.

\section{Drug Infusion}

Remifentanil (at a solution concentration of $10 \mu \mathrm{g} / \mathrm{ml}$ ) or saline was infused intravenously by a target controlled infusion (TCI) pump (Graseby 3500 TCI incorporating 'Diprifusor', SIMS Graseby Ltd, UK, supplied, preprogrammed with pharmacokinetic software, by Anaesthesia Technology Ltd, UK (Gray and Kenny, 1998)). The TCI pump controlled delivery of the remifentanil to achieve and maintain the desired remifentanil blood plasma concentration, based on the subject's sex, age, weight, and height incorporated into the existing published three-compartment pharmacokinetic model of remifentanil employed by the pump (Minto et al, 1997a,b). Subjects were blinded to the infusion of remifentanil or saline. Figure 1 illustrates the expected blood plasma concentration profile of remifentanil estimated from the existing pharmacokinetic model. This includes a sharp rise in plasma concentration at the start of infusion due to a loading dose administered by the TCI pump. The remifentanil plasma concentration profile is expected to differ from the remifentanil effect site concentration profile governing the fMRI response. The maximum plasma concentration of $1 \mathrm{ng} / \mathrm{ml}$ has previously been found to give a clear analgesic response to our painful thermal stimulus without excessive sedation or blood oxygen desaturation (Tracey et al, 2000b).

Seven thermal stimuli preceded the infusion. Infusion began and continued while stimuli 8-17 were delivered during an estimated constant plasma concentration of remifentanil. Infusion was halted and remifentanil concentration decayed from the action of esterases in the blood plasma while stimuli 18-28 were delivered (Figure 1).

\section{Image Analysis to Reveal Pain-Related Brain Activity}

Representative group maps of pain-related activity were calculated. These were used to confirm the existence of an appropriate anatomical region, responsive to pain, for use in the further analysis of the time dependence of the drug effect.

Image analysis to reveal significant brain activity based on changes in BOLD signal was performed on each subject's data using FMRIB Expert Analysis Tool (FEAT, http:// www.fmrib.ox.ac.uk/fsl) (Smith et al, 2001). The following processing was applied to each subject's time-series of fMRI volumes: motion correction using MCFLIRT (Bannister and Jenkinson, 2001; Jenkinson and Smith, 2001); spatial smoothing using a Gaussian kernel of full-width-halfmaximum $5 \mathrm{~mm}$; nonlinear highpass temporal filtering (Gaussian-weighted least-squares straight line fitting, with a high-pass filter cutoff of $70 \mathrm{~s}$ ) and subtraction of the mean of each voxel time-course from that time-course. The fMRI signal was then linearly modelled on a voxel by voxel basis using FMRIB's Improved Linear Model (FILM) with local autocorrelation correction (Woolrich et al, 2001).

Each thermal stimulus was assumed to elicit a hemodynamic response described by the convolution of a square on-off function of $3 \mathrm{~s}$ duration with a generalized hemodynamic response function (gamma variate function). The significance of the model fit to each voxel time-series was calculated, yielding statistical parametric maps of painrelated activity for each subject and infusion. These were combined using FEAT in a second level random-effects group analysis following the coregistration of each scan to the standard of the MNI (Montreal Neurological Institute). This resulted in a $Z$-score map of statistically significant pain-related activity across all infusions (Figure 2). All infusions were included as this improved the power to detect pain-related activity. A cluster-based correction of the $Z$-statistic images was performed. The raw $Z$-statistic images from the group analysis were thresholded at $Z$ scores greater than 4.0. This conservative threshold was chosen to preserve anatomical clarity in the display of the brain activity maps (Figure 2). For each resulting cluster of spatially connected voxels surviving the $Z$ threshold, a cluster probability threshold of $P=0.01$ was applied to the computed significance of that cluster. The cluster-based significance thresholding at $P<0.01$ provides a correction for the problem of multiple comparisons. The technique of cluster-based significance thresholding is described further

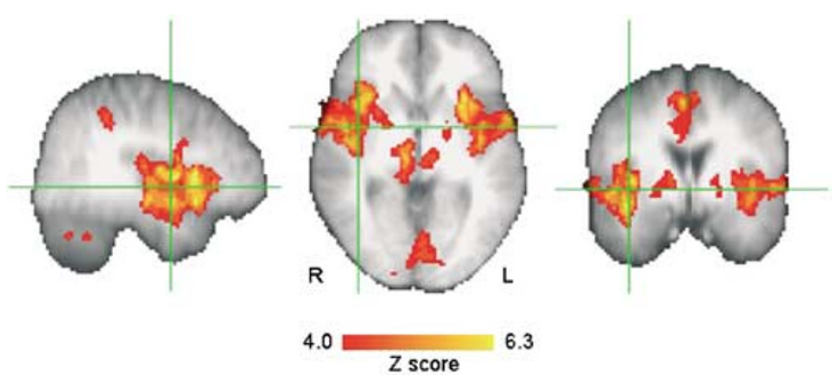

Figure 2 Representative group activity in response to painful thermal stimuli. Random effects group analysis from 18 datasets, group $Z>4.0$ and cluster threshold $P<0.01$. The background gray-scale image is the mean structural scan of all subjects after each scan was coregistered to the standard of the MNI. The cross-hairs indicate the right-sided insular cortex contralateral to the stimulus. This anatomical region was used in further analysis of the $\mathrm{fMRl}$ time-series to estimate the pharmacokineticpharmacodynamic parameters of remifentanil. 
by Forman et al (1995), Friston et al (1994), and Worsley et al (1992).

A previous fMRI study of steady-state infusion of remifentanil has shown pain-related activity to be robust in the insular cortices and significantly modulated by the drug (Tracey et al, 2000b; Tracey, 2001). The right-sided insular cortex contralateral to the stimulus was therefore chosen for further time-series analysis, aimed not simply at detecting that modulation but aimed at describing its timecourse. The boundaries of the insular cortex contralateral to the stimulus were manually defined from the MNI standard brain. Following coregistration of the functional scans to this standard, the BOLD signal time-course of the contralateral insula was extracted for each individual for further analysis.

\section{Pharmacokinetic-Pharmacodynamic Parameters from fMRI}

We have described in a previous study (Wise et al, 2002) analysis of data acquired in the experimental protocol given above confirming that the existing pharmacokinetic-pharmacodynamic model describing the magnitude of drug effect, previously established by EEG measurements, could be used to identify the regions whose pain-related activity was modulated by remifentanil. Importantly, the study presented here takes a different approach with the specific aim of characterizing the time-course of drug effect within the insular region in a novel manner using fMRI, without use of the pharmacokinetic-pharmacodynamic time constants derived from EEG measures of drug effect (Minto et al, 1997a,b). This study therefore develops and demonstrates the methods that reveal the potential of fMRI for determining pharmacokinetic-pharmacodynamic parameters based on function within a specific brain region.

By examination of the fMRI time-series in the contralateral insula, parameters describing the time-of-action of remifentanil were estimated. A region of interest analysis was deemed necessary to achieve an adequate signal-tonoise ratio in the time-series data.

The characteristics of the hemodynamic response on and off remifentanil, during the different epochs of the experiment, were examined to identify a suitable measure of drug effect (pharmacodynamics) in the fMRI signal. The spatial average of each subject's fMRI time-series was calculated over the region of interest for each infusion. For remifentanil and separately saline infusions, this time-series was then averaged across stimuli and finally across subjects for epochs of stimulation preinfusion (stimuli 1-7), during infusion when plasma concentration was expected to be constant (stimuli 11-17) and during the washout period (18-28) (Figure 3). The primary drug-dependent parameter of the BOLD hemodynamic response to the stimuli appeared to be the response amplitude. A model was developed to describe this response amplitude for each stimulus from the fMRI signal.

The hemodynamic response to each stimulus was described by a separate regressor formed by convolving the input stimulus function with a generalized gamma variate hemodynamic response function of full-width at half-maximum $6 \mathrm{~s}$ (FMRIB Expert Analysis Tool, http:// www.fmrib.ox.ac.uk/fsl). The mean lag of the hemodynamic
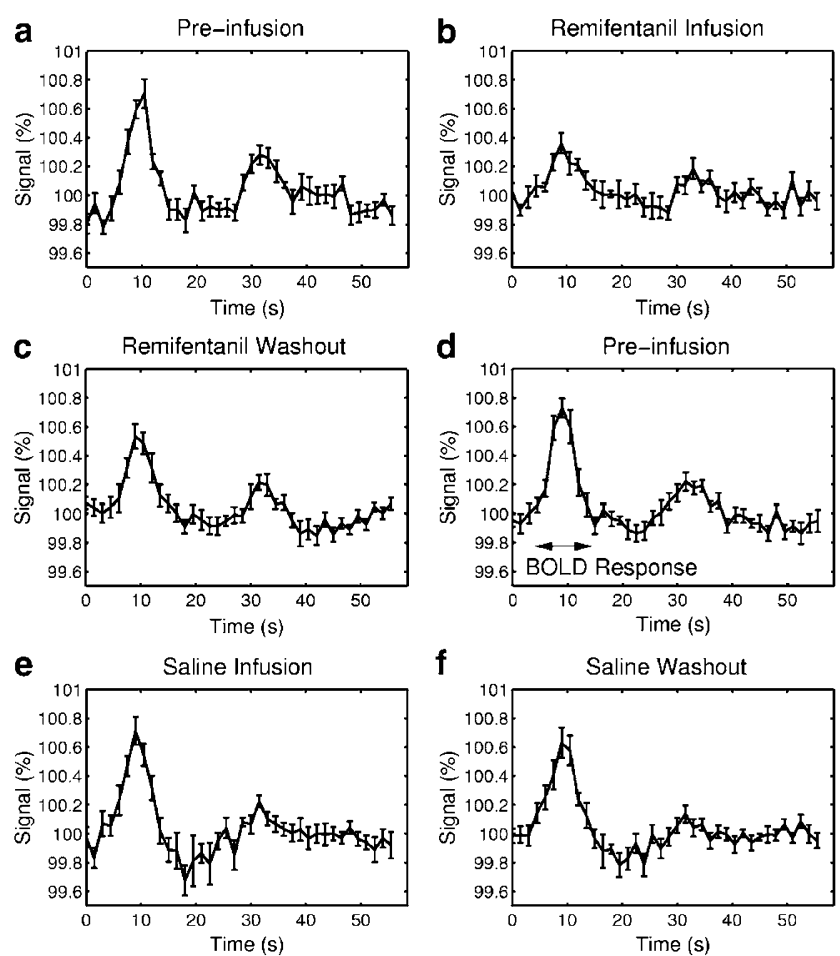

Figure 3 Mean $\mathrm{fMRI}$ signal over stimulus cycles and subjects in the insular region of interest following painful stimulation. The peak centered at a delay of approximately $9 \mathrm{~s}$, represents the BOLD response to the brief noxious stimulation. Time $=0 \mathrm{~s}$, indicates the start of the $3 \mathrm{~s}$ painful stimulus. Error bars indicate the SE over subjects. $(a-c)$ are derived from the remifentanil infusions, while $(d-f)$ are derived from the saline infusions. (a, d) are the mean $\mathrm{MMRI}$ signal over the first stimulus cycles I-7 during the preinfusion period. (b, e) are the mean $\mathrm{MMRI}$ signal over stimulus cycles I I17 during the steady infusion period ( $\mathrm{ng} / \mathrm{ml}$ estimated plasma concentration). (c, f) are the mean $\mathrm{fMRI}$ signal over stimulus cycles 18-28 during the remifentanil (or saline) washout period.

response function was chosen as $7.5 \mathrm{~s}$ after comparison with the group mean insular BOLD response to the painful stimulation, from the saline infusions only. This lag ensured a modeled response that peaked at the same time as the BOLD response to pain and provided a visually reasonable representation of it. Only the data from the saline infusions, and not the remifentanil infusions, was used to verify the suitability of the hemodynamic response function in order to reduce the possibility of overfitting the remifentanil data used for pharmacokinetic modeling. Least-squares linear regression between the insular time-series and the model was performed. Each regression coefficient therefore represented the amplitude of a BOLD response to pain.

To characterize the pharmacodynamic effects of remifentanil, BOLD response amplitude and perceived pain intensity were tested for a significant decrease between saline and remifentanil infusion sessions and between the two portions of the experiment (Figure 4): preinfusion (stimuli 1-7) and the period of steady infusion (stimuli 1117). Significance was tested with a paired one-tailed Student's $t$-test at $P<0.05$.

BOLD response amplitudes to each stimulus were pooled (averaged) across subjects to achieve adequate signal-tonoise for the parameter estimation (Figure 5). A further model was constructed to describe the temporal variation of 
a BOLD Response Amplitude

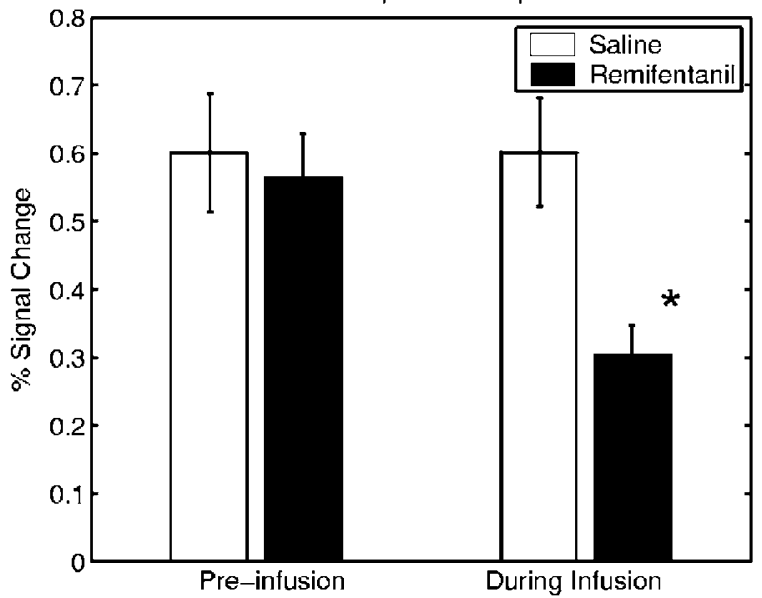

b

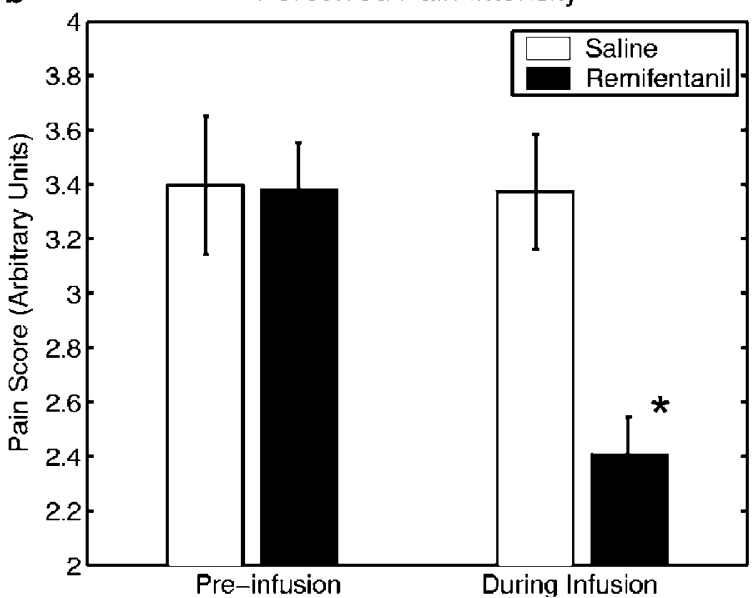

Figure $4 \mathrm{fMRl}$ measured brain activity and perceived pain intensity. Mean amplitudes of the BOLD response to pain (a) from the insular region of interest and mean perceived pain intensity scores (b) from blocks of painful stimulation preceding infusion (stimuli I-7) and during steady infusion (stimuli ||$-17$ ). Error bars indicate the SE of the mean across subjects. Filled bars indicate remifentanil infusion. Unfilled bars indicate saline infusion. *Indicates a significant reduction of BOLD amplitude and perceived pain intensity on infusion of remifentanil compared to preinfusion and also a significant reduction in these quantities during remifentanil infusion compared to saline infusion. Significance was tested with a paired one-tailed Student's t-test at $P<0.05$.

the BOLD response amplitudes for the remifentanil infusions only. Modeling of the time dependence of the BOLD amplitude and perceived pain intensity scores was not performed for the saline infusion because no significant change in amplitude of BOLD response or of pain scores was detected. For the purposes of estimating the time of action of remifentanil, the variation of BOLD response amplitude with remifentanil effect site concentration was assumed to be linear over the small range of concentrations applied.

Considering first the period of infusion of remifentanil and assuming a step change in remifentanil plasma concentration at $t_{\mathrm{I}}=0$ (Figure 1), the amplitude of the BOLD response to stimulation was described by

$$
S\left(t_{\mathrm{I}}\right)=S_{0}-\left(S_{0}-S_{\mathrm{I}}\right)\left[1-\exp \left(-k_{\mathrm{I}} t_{\mathrm{I}}\right)\right]
$$

where $k_{\mathrm{I}}$ is the plasma-BOLD effect equilibration rate constant and the associated half life of equilibration is given by $t_{1 / 2 \mathrm{I}}=\ln 2 / k_{\mathrm{I}} \cdot S_{0}$, the BOLD response amplitude at zero remifentanil concentration, was taken as the mean BOLD response amplitude for the stimuli 1-7 preceding remifentanil infusion. $t_{1 / 2 \mathrm{I}}$ was substituted into Eq. (1) and was estimated along with $S_{\mathrm{I}}$ (BOLD response amplitude at $1 \mathrm{ng} /$ $\mathrm{ml}$ effect site concentration) by fitting as described below. $S_{0}$ is shown in Figure 4, whereas $S_{\mathrm{I}}$ was estimated by fitting to be $0.27 \%$ signal change with $95 \%$ confidence intervals of 0.08 and $0.46 \%$.

Considering now the period, beginning at $t_{\mathrm{W}}=0$ (Figure 1), when remifentanil infusion was halted, the pain-related BOLD responses were modeled by the following decay function:

$$
S\left(t_{\mathrm{W}}\right)=S_{0}-\left(S_{0}-S_{\mathrm{W}}\right) \exp \left(-k_{\mathrm{W}} t_{\mathrm{W}}\right)
$$

where $S_{\mathrm{W}}$ was the BOLD response amplitude when infusion was halted, estimated from Eq. (1). $k_{\mathrm{W}}$ is the 'washout' rate constant and the associated washout half-life of remifentanil is given by $t_{1 / 2 \mathrm{~W}}=\ln 2 / k_{\mathrm{W}}$. This forms our estimate of the time taken for the effect of the remifentanil on the fMRI BOLD signal in the insular cortex to reduce by $50 \%$. $t_{1 / 2 \mathrm{~W}}$ was substituted into Eq. (2) and estimated by fitting.

The pharmacokinetic parameters were estimated by nonlinear least-squares fitting of the fMRI determined BOLD response amplitudes to Eqs. (1) and (2). Fitting was performed with a Gauss-Newton algorithm with Levenberg-Marquardt modifications for global convergence implemented using MATLAB software (The Mathworks, Inc., MA). The $95 \%$ confidence intervals were estimated on the parameters $t_{1 / 2 \mathrm{I}}$ and $t_{1 / 2 \mathrm{~W}}$ (Table 1).

To allow comparison of fMRI with an alternative pharmocodynamic effect of remifentanil, the same pharmacokinetic analysis was applied to group mean perceived pain intensity scores. Pain scores instead of BOLD response amplitudes were applied in Eqs. (1) and (2) (Figure 5) to estimate $t_{1 / 2 I}$ and $t_{1 / 2 W}$ (Table 1 ).

\section{RESULTS}

\section{Pain-Related Brain Activity}

Throughout any one imaging session, painful stimuli of $3 \mathrm{~s}$ duration were presented at a constant maximum temperature. The mean $\pm \mathrm{SD}$ was $56.4 \pm 1.4^{\circ} \mathrm{C}$, and stimulus temperature was applied across nine subjects and two sessions. A network of brain regions was observed to activate in response to the brief painful thermal stimuli (Figure 2). The group average map from a random effects analysis incorporates scans taken during remifentanil and saline infusions, from all subjects $(n=9)$. Regions including the bilateral insular cortices, the anterior cingulate cortex, the cerebellum, the bilateral secondary sensory region (SII) and the bilateral thalamus were active at this statistical level $(Z>4.0$ and activated cluster significance $P<0.01)$. This pattern of activity is consistent with previous reports of thermal pain-related brain activity (Becerra et al, 1999; Jones et al, 1991a; Peyron et al, 2000; Talbot et al, 1991; Tracey et al, 2000a). The right-sided insular cortex (contralateral to the stimulus), defined from the MNI standard brain, was chosen for further time-series analysis. 

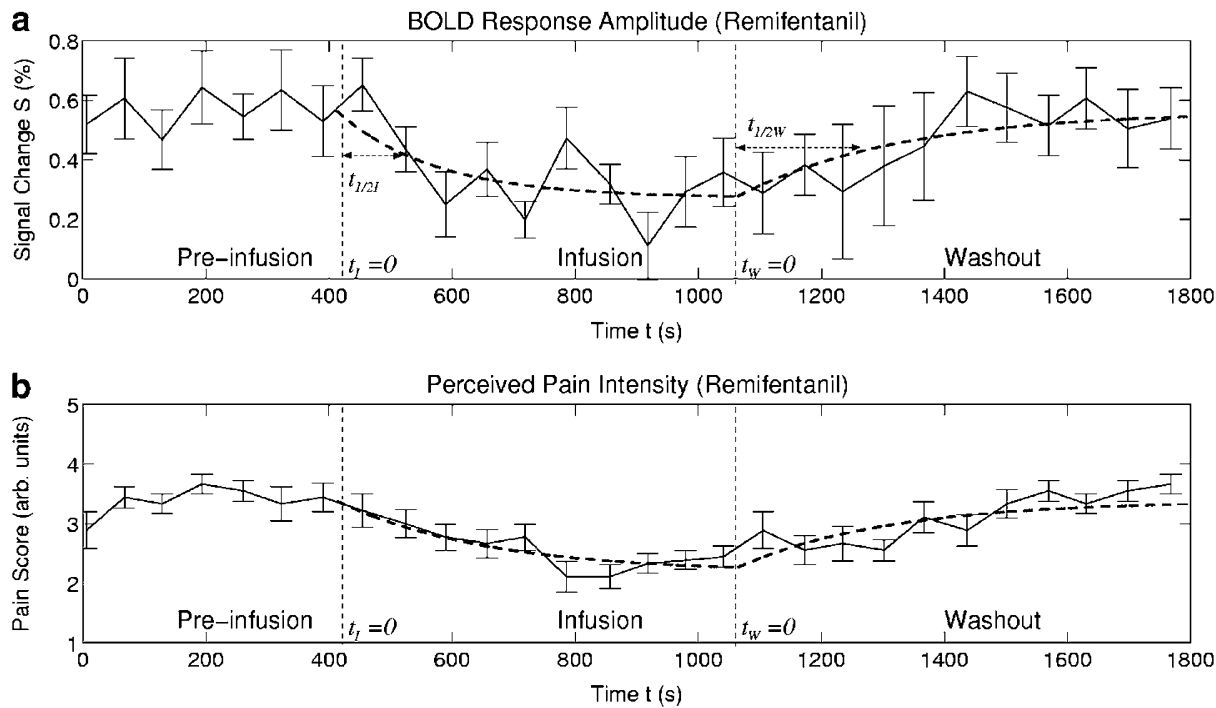

Figure 5 Time-course of $\mathrm{fMRI}$ and perceived pain intensity. Mean amplitude of the BOLD response to pain (solid line, a) and mean perceived pain intensity scores (solid line, b) during the course of the remifentanil sessions. Each point represents the response to one stimulus. Error bars indicate the SE of the mean across subjects. $t_{1}=0$ and $t_{w}=0$ indicate the onset and cessation of remifentanil infusion, respectively. The heavy broken lines indicate the fitted exponential models from which the onset equilibration half-life $\left(t_{1 / 21}\right)$ and the washout half-life of drug activity $\left(t_{1 / 2} w\right)$ were estimated.

Table I Pharmacokinetic-Pharmacodynamic Parameters of Remifentanil Derived from $\mathrm{MMR}$ BOLD Response Amplitude and Reported Pain Intensity

\begin{tabular}{lll}
\hline & $\begin{array}{c}\text { fMRI BOLD } \\
\text { response }\end{array}$ & $\begin{array}{c}\text { Perceived pain } \\
\text { intensity }\end{array}$ \\
\hline Equilibration half-life $\left(t_{1 / 21}\right)(\mathrm{min})$ & $1.83(-2.17,5.83)$ & $2.80(-0.12,5.73)$ \\
'Washout' half-life $\left(t_{1 / 2 W}\right)(\mathrm{min})$ & $3.07(1.09,5.05)$ & $2.80(1.12,4.48)$ \\
\hline
\end{tabular}

Figures in parentheses indicate $95 \%$ confidence intervals for the estimated parameters.

Of the regions listed, this region shows significant modulation of pain-related activity resulting from remifentanil infusion (Tracey, 2001; Wise et al, 2002), and is observed to be one of the most robustly pain-active regions (Peyron et al, 2000). These factors would suggest that, given the limited signal-to-noise ratio of drug-induced changes in the fMRI signal, the likelihood of extracting meaningful pharmacokinetic data on drug effect was highest for this chosen region of interest.

\section{Hemodynamic Response}

It was necessary to establish an appropriate pharmacodynamic measure (a measure of drug effect) from within the fMRI signal. Figure 3 illustrates the average form of the hemodynamic BOLD response in the right insular region of interest, for stimuli within the three epochs of the experiment: preinfusion, steady infusion and washout. FMRI signal samples are spaced apart by $1 / 2 \mathrm{TR}=1.5 \mathrm{~s}$, made possible by jittering the stimulus onset with respect to slice acquisition timing. The time scale (abscissa) covers approximately one interstimulus period.

The consistent signal peak at approximately $9 \mathrm{~s}$ after the onset of the painful stimulation represents the principal hemodynamic response to the thermal stimulus. The second smaller peak at about $32 \mathrm{~s}$ after the start of the painful stimulus may arise from the pain intensity rating and subsequent button-press. This is consistent with the insula's established role in somatosensory processing (Burton et al, 1993), involvement in non-nociceptive touch (JohansenBerg et al, 2000) and also previously reported activity during button pressing (Johansen-Berg and Matthews, 2002).

The form of the hemodynamic response during saline infusions (Figure $3 \mathrm{~d}-\mathrm{f}$ ) remains similar throughout the course of the imaging session. The amplitude of response is consistent (approximately $0.6 \%$ ) and the width (full-width at half-maximum) appears consistent across epochs. However, the amplitude of the response appears reduced during remifentanil infusion (Figure $3 \mathrm{~b}$ ) compared to the preinfusion period (Figure 3a). The mean response during the washout period (Figure 3c) shows a recovery of amplitude. Figure 3 demonstrates the suitability of the BOLD response amplitude as a marker of the effect of remifentanil on painrelated brain activity. The amplitude was established by comparing the fMRI signal after each stimulus to a hemodynamic response function.

\section{Time Dependence of Pharmacological Effects}

To identify a drug effect, BOLD response amplitude and perceived pain intensity were compared during two portions of the experiment (Figure 4): preinfusion (stimuli 1-7) and during the period of steady infusion (stimuli 1117) having allowed several minutes for a period of bloodbrain equilibration (Figure 1). The mean BOLD response amplitude and the reported pain intensity arising from stimulation were not significantly altered by saline infusion. However, remifentanil infusion significantly decreased BOLD response amplitude and perceived pain intensity within session and also in comparison to saline infusion 
$(P<0.05$, Figure 4$)$. Remifentanil at an estimated $1 \mathrm{ng} / \mathrm{ml}$ plasma concentration reduced BOLD response amplitude by approximately half on average in the insula.

The pharmacokinetic model of remifentanil predicted a step change in blood plasma concentration of the drug at the start of infusion $\left(t_{\mathrm{I}}=0\right.$, Figure 1$)$, followed by a period of steady plasma concentration. We would expect a lag between the increase in plasma concentration and the effect of the drug, governed by its concentration at the effect site, assumed to be in the brain. This lag is conventionally described by a blood plasma-effect site equilibration rate constant $\left(k_{\mathrm{e} 0}\right)$ and associated half-life of equilibration $\left(t_{1 / 2} k_{\mathrm{e} 0}=\ln 2 / k_{\mathrm{e} 0}\right)$ (Figure 1$)$. This time constant represents the time of onset of action of the drug. We suggest that the effect of the equilibration time is evident in the fMRI signal. An fMRI-based group-mean equilibration time that we will refer to as $t_{1 / 2}$, was estimated (Table 1 ) from the temporal profile of decrease in the BOLD response amplitudes, characterized by a single exponential function (Figure 5), following the commencement of infusion.

Following the end of infusion at $1064 \mathrm{~s}$ into the imaging session $\left(t_{\mathrm{W}}=0\right.$, Figure 1$)$, the effect of remifentanil was expected to decay with what is often termed the contextsensitive half-life (Hughes et al, 1992). A half-life of drug activity may be defined based on the fMRI signal and in this paper is labeled by $t_{1 / 2 \mathrm{~W}}$ (a pharmacodynamic contextsensitive half-life). During the 'washout' period, we assumed a simple exponential recovery of the BOLD response amplitude towards the amplitude estimated during the preinfusion period (Figure 5). In this way, $t_{1 / 2 \mathrm{~W}}$ was estimated (Table 1) as a group-mean pharmacokineticpharmacodynamic parameter based on changes in brain function within a specific region of brain (the insula).

Since there was no significant modulation of the BOLD response amplitude or perceived pain intensity by saline infusion, it was not possible to estimate the blood-effect site equilibration half-life and washout half-life for saline.

Group-mean perceived pain intensities followed a similar time-course to the changes in pain-related BOLD response amplitude in the contralateral insular region of interest (Figure 5) (correlation coefficient $r=0.65, P<0.01$ ). Figure 5 shows that these time-courses are qualitatively similar to the profile of the estimated effect site concentration of remifentanil predicted from the exisiting pharmacokineticpharmacodynamic model (Minto et al, 1997a,b) (Figure 1). The parameters $t_{1 / 2 \mathrm{I}}$ and $t_{1 / 2 \mathrm{~W}}$ were estimated (Figure 5, Table 1) from the temporal variation in pain intensity scores, used as a marker of drug effect, by the same approach as adopted for the analysis of BOLD response amplitude.

\section{DISCUSSION}

We have determined using fMRI the times of onset and offset of remifentanil's action. To our knowledge this is the first use of fMRI to establish pharmacokinetic-pharmacodynamic parameters based on drug-induced changes in task-related brain function. We have previously demonstrated using fMRI the dose dependence and specificity of remifentanil's effect in reducing pain-related activity (Tracey et al, 2000b; Tracey, 2001). We have further investigated regional modulation of pain-related brain activity by employing the pre-existing pharmacokineticpharmacodynamic model of the remifentanil effect site (brain) concentration in the analysis of fMRI data (Wise et al, 2002). The present study extends this work considerably by estimating the time-course of the pharmacodynamic effect in the brain from the fMRI data under the assumption of known pharmacokinetics of blood plasma concentration at a spatial resolution that is meaningful in terms of targeted drug discovery developments.

One brain region, activated by the noxious stimulus, was selected for analysis of time-varying drug activity: the contralateral (right sided) insula. In addition to showing opioid-induced modulation of fMRI-measured activity, the insular is consistently one of the most significantly painactive regions in this experiment and in previous studies (Peyron et al, 2000). Detection of drug modulation of pain activity is most likely where the stimulus-related activity is initially most significant. Furthermore, the posterior insula has recently been implicated as a thermosensory cortex (Craig et al, 2000). Peyron et al (1999) suggested the role of insula in the sensory discriminative dimension of pain intensity encoding. PET ligand binding studies have shown the insula to posses a high concentration of $\mu$-opioid receptors (Jones et al, 1991b,c), suggesting the insula as a potential site of analgesic action for remifentanil, a $\mu$-opioid agonist. It was therefore an ideal anatomical region to select for our pharmacokinetic-pharmacodynamic modeling. The change in amplitude of the stimulus-induced BOLD response proved to be a sensitive pharmacodynamic marker with a halving of amplitude by the infusion of $1 \mathrm{ng} / \mathrm{ml}$ plasma concentration of remifentanil. The sensitivity of fMRI to a given dose of remifentanil is greater than for EEG spectral edge measurements. A concentration of $11.2 \mathrm{ng} / \mathrm{ml}$, which is larger than typically used clinically, was required to provide a $50 \%$ decrease of the spectral edge frequency (Minto et al, 1997a). The concentration adopted in this study of $1 \mathrm{ng} / \mathrm{ml}$ is at the low end of the clinical dose range. fMRI may therefore be more appropriate tool for examining the cerebral effects of clinically relevant doses.

The BOLD response amplitude followed a similar temporal profile to the behavioral measures of perceived pain intensity. The simultaneous variation of remifentanil concentration, perceived pain intensity and BOLD response amplitude, and the differences in these quantities between remifentanil and saline infusions, suggest that changes in the BOLD signal reflect the analgesic effect of remifentanil on pain-related brain activity. The experiments do not prove that remifentanil is acting selectively and only at the neuronal level within the insula. Vascular effects of opioids may occur although a global hemodynamic confound is unlikely as our previous study revealed that brain activity in the occipital cortex arising from a visual stimulus did not exhibit a remifentanil dose-dependent effect (Tracey et al, 2000b; Tracey, 2001). Neuronal opioid effects may also occur at a lower level in the brainstem, spinal cord and periphery (Lorenz et al, 2000; Wagner et al, 2001). However, we believe based upon our previous work that the major effect we are measuring is likely to result from the direct action of remifentanil at the insular site.

The pain-related BOLD response amplitude depends on the concentration of remifentanil at the site of drug action, 
often termed the effect site concentration. Existing pharmacokinetic-pharmacodynamic relationships for drugs such as remifentanil (Egan, 1995; Patel and Spencer, 1996) often express the characteristic times of onset or offset of drug action in terms of the temporal variation in the notional effect site concentration. That variation is studied via a pharmacodynamic effect, for example EEG spectral changes, that is related to the effect site concentration by a model (Shargel and Yu, 1999). However, in this study, we have directly examined the temporal variation of the pharmacodynamic effects on the BOLD signal and the perceived pain intensity. We have adopted the same easily implemented nonlinear model for the time dependence of the perceived pain intensity and the BOLD response, described by a single amplitude parameter for each stimulus. While a nonlinear model could be used to describe the complete fMRI signal, this would be more complex to implement and our consistent and simple approach to the fMRI and behavioral data allows us to easily compare the time dependence of pain intensity and an fMRI index of brain activity.

We have characterized the drug effects by a half-life of onset $\left(t_{1 / 2 \mathrm{I}}\right)$ and offset $\left(t_{1 / 2 \mathrm{~W}}\right)$ (Table 1) of changes in the BOLD signal and reported pain intensity. Under the assumption, supported by the present study, of a linear relationship between the change in BOLD response amplitude or reported pain intensity and the remifentanil effect site concentration, these functionally defined halflives are equivalent to the equilibration half-life $\left(t_{1 / 2} k_{\mathrm{e} 0}\right)$ and context sensitive half-life $\left(t_{1 / 2}\right)$ for effect site concentration established in previous studies of remifentanil.

The plasma-effect site (brain) equilibration half-life $t_{1 / 2} k_{\mathrm{e} 0}(\mathrm{~min})$ (Figure 1), describing the onset delay, has been estimated from EEG measurements as $1.03 \mathrm{~min}$ (Minto et al, 1997a), $1.41+1.48 \mathrm{~min}$ (Egan et al, 1994), $1.23+0.96 \mathrm{~min}$ (Hermann et al, 1999) and $1.6 \pm 0.9$ min (Egan et al, 1996). The ventilatory response to carbon dioxide has estimated the equilibration half-life to be $2.04 \mathrm{~min}(-0.24,4.32 \mathrm{~min}$ : 95\% confidence intervals) (Babenco et al, 2000). Analgesic measurements have estimated $1.31 \pm 1.5 \mathrm{~min}$ (Glass et al, 1993) These previous estimates by alternative techniques are in agreement with our estimates of $t_{1 / 2 \mathrm{I}}=1.83 \mathrm{~min}$ $(-2.17,5.83 \mathrm{~min}$ : $95 \%$ confidence intervals) from the insular cortical BOLD response and $t_{1 / 2 \mathrm{I}}=2.80 \mathrm{~min} \quad(-0.12$, $5.73 \mathrm{~min}$ : $95 \%$ confidence intervals) for reported pain intensity.

The characteristic time of offset of action or contextsensitive half-life $\left(t_{1 / 2}\right)$ (Hughes et al, 1992) is the time taken for the effect site concentration to fall by $50 \%$ following a variable length infusion. Owing to the rapid breakdown of remifentanil in the blood and tissues, this half-life does not vary with the duration of infusion (Minto et al, 1997a,b). EEG measurements have yielded a context-sensitive half-life of 3-5 min (estimated at $3.7 \mathrm{~min}$ from the population model, for volunteers in this study) (Minto et al, 1997b), in agreement with our fMRI estimate from the insular cortex $t_{1 / 2 \mathrm{~W}}=3.07 \mathrm{~min}(1.09,5.05 \mathrm{~min}: 95 \%$ confidence intervals $)$, and from reported pain intensity $t_{1 / 2 \mathrm{~W}}=2.80 \mathrm{~min}(1.12$, 4.48 min: 95\% confidence intervals). Half-life measurements based on concentration data give $3.2 \pm 0.9$ min (Kapila et al, 1995) and based on a pharmacodynamic measure of ventilation give $5.4 \pm 1.8 \mathrm{~min}$ (Kapila et al, 1995).
In our analysis to yield characteristic times of action for remifentanil, we have assumed plasma remifentanil concentrations predicted from previously published pharmacokinetic data (Minto et al, 1997a), rather than measuring remifentanil concentration directly. However, given linear superposition of doses under first-order pharmacokinetics, estimates of half-lives would be little affected by a scaling of concentration between volunteers. Furthermore, the unwanted influence of interindividual variability in actual plasma levels was likely reduced by the use of pooled data from the sample population to perform modeling. The agreement, within estimated confidence intervals, of our pharmacokinetic parameters with previous measurements from other techniques, supports our simple fMRI-pharmacokinetic modeling procedure. However, the confidence and precision of our pharmacokinetic estimates might be improved by using a noxious stimulus for which a shorter interstimulus interval is possible, hence improving the time resolution.

Ventilatory changes and analgesia that have been used to characterize remifentanil in past studies are clinically relevant pharmacodynamic effects although they give no information about where the neural correlates of analgesia may occur. The clinical relevance of the change in spectral characteristics of the EEG arising from cortical neurons, on infusion of remifentanil, however is less clear. Unlike EEG spectral measurements, the fMRI data presented in this study relates specifically to the processing of pain signals that can be correlated with reported pain perception. Furthermore, the spatial localization and depth of penetration of EEG is poor compared to fMRI. Unlike EEG, fMRI has the potential to differentiate and visualize the influence of pharmacological agents on small brain structures, deep brain structures and hidden cortical areas such as the insular cortex. While PET is capable of revealing drug receptor occupancy, fMRI is better suited for studying complex paradigms such as those involving pain that may be modulated by drugs. The potential of fMRI for identifying the temporal dynamics of drug activity through clinically relevant changes in regional brain function has been demonstrated. Further experimentation to compare the temporal behavior of pain-related brain activity across many regions with subtle changes in pain perception may reveal those regions most critical for drug-induced analgesia.

\section{ACKNOWLEDGEMENTS}

We acknowledge the generous support of The Dr Hadwen Trust for Humane Research, The Wellcome Trust (RGW, Advanced Training Fellowship Grant Code 067037/Z/02/Z), GlaxoSmithKline (PW), UK Medical Research Council and Higher Education Funding Council (IT).

\section{REFERENCES}

Babenco HD, Conard PF, Gross JB (2000). The pharmacodynamic effect of a remifentanil bolus on ventilatory control. Anesthesiology 92: 393-398.

Bannister PR, Jenkinson M (2001). Robust affine motion correction in fMRI time series. Neuroimage 13: S70. 
Becerra LR, Breiter HC, Stojanovic M, Fishman S, Edwards A, Comite AR et al (1999). Human brain activation under controlled thermal stimulation and habituation to noxious heat: an fMRI study. Magn Reson Med 41: 1044-1057.

Bloom AS, Hoffmann RG, Fuller SA, Pankiewicz J, Harsch HH, Stein EA (1999). Determination of drug-induced changes in functional MRI signal using a pharmacokinetic model. Hum Brain Mapp 8: 235-244.

Breiter HC, Gollub RL, Weisskoff RM, Kennedy DN, Makris N, Berke JD et al (1997). Acute effects of cocaine on human brain activity and emotion. Neuron 19: 591-611.

Burton H, Videen TO, Raichle ME (1993). Tactile-vibrationactivated foci in insular and parietal-opercular cortex studied with positron emission tomography: mapping the second somatosensory area in humans. Somatosens Mot Res 10: 297-308.

Coull JT, Nobre AC, Frith CD (2001). The noradrenergic $\alpha 2$ agonist clonidine modulates behavioural and neuroanatomical correlates of human attentional orienting and alerting. Cerebral Cortex 11: 73-84.

Craig AD, Chen K, Bandy D, Reiman EM (2000). Thermosensory activation of insular cortex. Nat Neurosci 3: 184-190.

Egan TD (1995). Remifentanil pharmacokinetics and pharmacodynamics. A preliminary appraisal. Clin Pharmacokinet 29: 80-94.

Egan TD, Minto CF, Hermann DJ, Barr J, Muir KT, Shafer SL (1996). Remifentanil versus alfentanil: comparative pharmacokinetics and pharmacodynamics in healthy adult male volunteers. Anesthesiology 84: 821-833.

Egan TD, Minto CF, Lemmens HJ, Muir KT, Hermann D, Shafer SL (1994). Remifentanil versus alfentanil: comparative pharmacodynamics. Anesthesiology 81: A374.

Forman SD, Cohen JD, Fitzgerald M, Eddy WF, Mintun MA, Noll DC (1995). Improved assessment of significant activation in functional magnetic resonance imaging (fMRI): use of a clustersize threshold. Magn Reson Med 33: 636-647.

Friston KJ, Worsley KJ, Frackowiak RS, Maziotta JC, Evans AC (1994). Assessing the significance of focal activations using their spatial extent. Hum Brain Mapp 1: 214-220.

Furey ML, Pietrini P, Haxby JV (2000). Cholinergic enhancement and increased selectivity of perceptual processing during working memory. Science 290: 2315-2319.

Glass PS, Hardman D, Kamiyama Y, Quill TJ, Marton G, Donn KH et al (1993). Preliminary pharmacokinetics and pharmacodynamics of an ultra-short-acting opioid: remifentanil (GI87084B). Anesth Analg 77: 1031-1040.

Gray JM, Kenny GN (1998). Development of the technology for 'Diprifusor' TCI systems. Anaesthesia 53: 22-27.

Hariri AR, Mattay VS, Tessitore A, Fera F, Smith WG, Weinberger DR (2002). Dextroamphetamine modulates the response of the human amygdala. Neuropsychopharmacology 27: $1036-1040$.

Hermann DJ, Egan TD, Muir KT (1999). Influence of arteriovenous sampling on remifentanil pharmacokinetics and pharmacodynamics. Clin Pharmacol Ther 65: 511-518.

Hoke JF, Cunningham F, James MK, Muir KT, Hoffman WE (1997). Comparative pharmacokinetics and pharmacodynamics of remifentanil, its principle metabolite (GR90291) and alfentanil in dogs. J Pharmacol Exp Ther 281: 226-232.

Honey GD, Bullmore ET, Soni W, Varatheesan M, Williams SC, Sharma T (1999). Differences in frontal cortical activation by a working memory task after substitution of risperidone for typical antipsychotic drugs in patients with schizophrenia. Proc Natl Acad Sci USA 96: 13432-13437.

Hughes MA, Glass PS, Jacobs JR (1992). Context-sensitive halftime in multicompartment pharmacokinetic models for intravenous anesthetic drugs. Anesthesiology 76: 334-341.
Jenkinson M, Smith S (2001). A global optimisation method for robust affine registration of brain images. Med Image Anal 5: 143-156.

Jensen MP, Karoly P (2001). Self Report Scales and Procedures for Assessing Pain in Adults. In: Turk DC, Elzack R (eds). Handbook of Pain Assessment. Guildford Press: New York. pp 15-34.

Johansen-Berg $\mathrm{H}$, Christensen V, Woolrich M, Matthews PM (2000). Attention to touch modulates activity in both primary and secondary somatosensory areas. Neuroreport 11: 1237-1241.

Johansen-Berg H, Matthews PM (2002). Attention to movement modulates activity in sensori-motor areas, including primary motor cortex. Exp Brain Res 142: 13-24.

Jones AK, Brown WD, Friston KJ, Qi LY, Frackowiak RS (1991a). Cortical and subcortical localization of response to pain in man using positron emission tomography. Proc R Soc Lond B Biol Sci 244: 39-44.

Jones AK, Friston KJ, Qi LY, Harris M, Cunningham VJ, Jones T et al (1991b). Sites of action of morphine in the brain. Lancet 338: 825.

Jones AK, Qi LY, Fujirawa T, Luthra SK, Ashburner J, Bloomfield P et al (1991c). In vivo distribution of opioid receptors in man in relation to the cortical projections of the medial and lateral pain systems measured with positron emission tomography. Neurosci Lett 126: 25-28.

Kapila A, Glass PS, Jacobs JR, Muir KT, Hermann DJ, Shiraishi M et al (1995). Measured context-sensitive half-times of remifentanil and alfentanil. Anesthesiology 83: 968-975.

Kimberg DY, Aguirre GK, Lease J, D’Esposito M (2001). Cortical effects of bromocriptine, a D-2 dopamine receptor agonist, in human subjects, revealed by fMRI. Hum Brain Mapp 12: 246-257.

Kleinschmidt A, Bruhn H, Kruger G, Merboldt KD, Stoppe G, Frahm J (1999). Effects of sedation, stimulation, and placebo on cerebral blood oxygenation: a magnetic resonance neuroimaging study of psychotropic drug action. NMR Biomed 12: 286-292.

Leslie RA, James MF (2000). Pharmacological magnetic resonance imaging: a new application for functional MRI. Trends Pharmacol Sci 21: 314-318.

Lorenz IH, Kolbitsch C, Schocke M, Kremser C, Zschiegner F, Hinteregger $\mathrm{M}$ et al (2000). Low-dose remifentanil increases regional cerebral blood flow and regional cerebral blood volume, but decreases regional mean transit time and regional cerebrovascular resistance in volunteers. Br J Anaesth 85: 199-204.

Malizia AL, Gunn RN, Wilson SJ, Waters SH, Bloomfield PM, Cunningham VJ et al (1996). Benzodiazepine site pharmacokinetic/pharmacodynamic quantification in man: direct measurement of drug occupancy and effects on the human brain in vivo. Neuropharmacology 35: 1483-1491.

Mattay VS, Callicott JH, Bertolino A, Heaton I, Frank JA, Coppola $\mathrm{R}$ et al (2000). Effects of dextroamphetamine on cognitive performance and cortical activation. Neuroimage 12: 268-275.

Minto CF, Schnider TW, Egan TD, Youngs E, Lemmens HJ, Gambus PL et al (1997a). Influence of age and gender on the pharmacokinetics and pharmacodynamics of remifentanil. I. Model development. Anesthesiology 86: 10-23.

Minto CF, Schnider TW, Shafer SL (1997b). Pharmacokinetics and pharmacodynamics of remifentanil. II. Model Application. Anesthesiology 86: 24-33.

Pappata S, Tavitian B, Traykov L, Jobert A, Dalger A, Mangin JF et al (1996). In vivo imaging of human cerebral acetylcholinesterase. J Neurochem 67: 876-879.

Patel SS, Spencer CM (1996). Remifentanil. Drugs 52: 417-427.

Petrovic P, Kalso E, Petersson KM, Ingvar M (2002). Placebo and opioid analgesia- imaging a shared neuronal network. Science 295: 1737-1740.

Peyron R, Garcia-Larrea L, Gregoire MC, Costes N, Convers P, Lavenne $\mathrm{F}$ et al (1999). Haemodynamic brain responses to acute 
pain in humans: sensory and attentional networks. Brain 122: $1765-1780$.

Peyron R, Laurent B, Garcia-Larrea L (2000). Functional imaging of brain responses to pain. A review and meta-analysis (2000). Neurophysiol Clin 30: 263-288.

Shargel L, Yu ABC (1999). Applied Biopharmaceutics and Pharmacokinetics. Appleton and Lange: Stamford.

Smith S, Bannister PR, Beckmann C, Brady JM, Clare S, Flitney D et al (2001). FSL: new tools for functional and structural brain image analysis. Neuroimage 13: S249.

Stein EA (2001). FMRI: a new tool for the in vivo localization of drug actions in the brain. J Anal Toxicol 25: 419-424.

Stein EA, Pankiewicz J, Harsch HH, Cho JK, Fuller SA, Hoffmann RG et al (1998). Nicotine-induced limbic cortical activation in the human brain: a functional MRI study. Am J Psychiatry 155: 1009-1015.

Talbot JD, Marrett S, Evans AC, Meyer E, Bushnell MC, Duncan $\mathrm{GH}$ (1991). Multiple representations of pain in human cerebral cortex. Science 251: 1355-1358.

Thiel CM, Henson RN, Dolan RJ (2002). Scopolamine but not lorazepam modulates face repetition priming: a psychopharmacological fMRI study. Neuropsychopharmacology 27: 282-292.

Thiel CM, Henson RNA, Morris JS, Friston KJ, Dolan RJ (2001). Pharmacological modulation of behavioural and neuronal correlates of repetition priming. J Neurosci 21: 6846-6852.
Tracey I (2001). Prospects for human pharmacological functional magnetic resonance imaging (phMRI). J Clin Pharmacol 41: 21S-28S.

Tracey I, Becerra L, Chang I, Breiter H, Jenkins L, Borsook D et al (2000a). Noxious hot and cold stimulation produce common patterns of brain activation in humans: a functional magnetic resonance imaging study. Neurosci Lett 288: 159-162.

Tracey I, Hicks G, Rogers R, Clare S, Bountra C, Barrington P et al (2000b). Pharmacological modulation of the central pain response. Proc Intl Soc Mag Reson Med 8: 913.

Wagner KJ, Willoch F, Kochs EF, Siessmeier T, Tolle TR, Schwaiger $M$ et al (2001). Dose-dependent regional cerebral blood flow changes during remifentanil infusion in humans: a positron emission tomography study. Anesthesiology 94: 732-739.

Wise RG, Rogers R, Painter D, Bantick S, Ploghaus A, Williams P et al (2002). Combining fMRI with a pharmacokinetic model to determine which brain areas activated by painful stimulation are specifically modulated by remifentanil. Neuroimage 16: 999-1014.

Woolrich M, Ripley B, Brady JM, Smith S (2001). Temporal autocorrelation in univariate linear modelling of fMRI data. Neuroimage 14: 1370-1386.

Worsley KJ, Evans AC, Marrett S, Neelin P (1992). A threedimensional statistical analysis for $\mathrm{CBF}$ activation studies in human brain. J Cereb Blood Flow Metab 12: 900-918. 